\title{
On Lacunary Mean Ideal Convergence in Generalized Random $n$-Normed Spaces
}

\author{
Awad A. Bakery ${ }^{1,2}$ and Mustafa M. Mohammed ${ }^{1,3}$ \\ ${ }^{1}$ Department of Mathematics, Faculty of Science and Arts, King Abdulaziz University (KAU), P.O. Box 80200, \\ Khulais 21589, Saudi Arabia \\ ${ }^{2}$ Department of Mathematics, Faculty of Science, Ain Shams University, P.O. Box 1156, Abbassia, Cairo 11566, Egypt \\ ${ }^{3}$ Department of Statistics, Faculty of Science, Sudan University of Science \& Technology, Khartoum, Sudan
}

Correspondence should be addressed to Awad A. Bakery; awad_bakery@yahoo.com

Received 6 January 2014; Accepted 17 March 2014; Published 24 April 2014

Academic Editor: Jianming Zhan

Copyright (C) 2014 A. A. Bakery and M. M. Mohammed. This is an open access article distributed under the Creative Commons Attribution License, which permits unrestricted use, distribution, and reproduction in any medium, provided the original work is properly cited.

\begin{abstract}
An ideal $I$ is a hereditary and additive family of subsets of positive integers $\mathbb{N}$. In this paper, we will introduce the concept of generalized random $n$-normed space as an extension of random $n$-normed space. Also, we study the concept of lacunary mean $(L)$-ideal convergence and $L$-ideal Cauchy for sequences of complex numbers in the generalized random $n$-norm. We introduce $I_{L}$-limit points and $I_{L}$-cluster points. Furthermore, Cauchy and $I_{L}$-Cauchy sequences in this construction are given. Finally, we find relations among these concepts.
\end{abstract}

\section{Introduction}

The sets of natural numbers and complex numbers will be denoted by $\mathbb{N}$ and $\mathbb{C}$, respectively. Fast [1] and Steinhaus [2] independently introduced the notion of statistical convergence for sequences of real numbers, which is a generalization of the concept of convergence. The concept of statistical convergence is a very valuable functional tool for studying the convergence problems of numerical sequences through the concept of density. Afterward, several generalizations and applications of this concept have been presented by different authors (see [3-6]). Kostyrko et al. [7] presented a generalization of the concept of statistical convergence with the help of an ideal $I$ of subsets of the set of natural numbers $\mathbb{N}$, and more is studied in [8-11]. This concept of ideal convergence plays a fundamental role not only in pure mathematics but also in other branches of science concerning mathematics, mainly in information theory, computer science, dynamical systems, geographic information systems, and population modelling. Menger [12] generalized the metric axioms by associating a distribution function with each pair of points of a set. This system is called a probabilistic metric space. By using the concept of Menger, Šerstnev [13] introduced the concept of probabilistic normed spaces. It provides an important area into which many essential results of linear normed spaces can be generalized; see [14]. Later, Alsina et al. [15] presented a new definition of probabilistic normed space which includes the definition of normed space which includes the definition of Šerstnev as a special case. The concept of ideal convergence for single and double sequences of real numbers in probabilistic normed space was introduced and studied by Mursaleen and Mohiuddine [16]. Mursaleen and Alotaibi [17] studied the notion of ideal convergence for single and double sequences in random 2-normed spaces, respectively. For more details and linked concept, we refer to [18-26]. In $[27,28]$, Gähler introduced a gorgeous theory of 2-normed and $n$-normed spaces in the 1960s; we have studied these subjects and constructed some sequence spaces defined by ideal convergence in $n$-normed spaces [29, 30]. Another important alternative of statistical convergence is the notion of lacunary statistical convergence introduced by Fridy and Orhan [31]. Recently, Mohiuddine and Aiyub [4] studied lacunary statistical convergence by introducing the concept 
$\Theta$-statistical convergence in random 2-normed space. Their work can be considered as a particular generalization of the statistical convergence. In [32], Mursaleen and Mohiuddine generalized the idea of lacunary statistical convergence with respect to the intuitionistic fuzzy normed space, and Debnath [33] investigated lacunary ideal convergence in intuitionistic fuzzy normed linear spaces. Also, lacunary statistically convergent double sequences in probabilistic normed space were studied by Mohiuddine and Savaş in [34]. Jebril and Dutta [35] introduced the concept of random $n$-normed space. In this paper, we firstly give some basic definitions and properties of random $n$-normed space in Section 2. In Section 3, we define a new and interesting notion of generalized random $n$-normed spaces; convergent sequences in it are introduced and we provide some results on it. In Section 4, we study lacunary mean $(L)$-ideal convergence and $L$-ideal Cauchy for sequences of complex numbers in the generalized random n-norm. Finally, in Section 5, we introduce $I_{L}$-limit points and $I_{L}$-cluster points. Moreover, Cauchy and $I_{L}$-Cauchy sequences in this framework are given, and we find relations among these concepts.

\section{Definitions and Preliminaries}

For the reader's expediency, we restate some definitions and results that will be used in this paper.

The notion of statistical convergence depends on the density (asymptotic or natural) of subsets of $\mathbb{N}$.

Definition 1. A subset $E$ of $\mathbb{N}$ is said to have natural density $\delta(E)$ if

$$
\delta(E)=\lim _{n \rightarrow \infty} \frac{1}{n}|\{k \leq n: k \in E\}| \text { exists, }
$$

where $|E|$ denotes the cardinality of the set $E$.

Definition 2. A sequence $\left(x_{k}\right)$ is statistically convergent to $\ell$ if, for every $\varepsilon>0$,

$$
\delta\left(\left\{k \in \mathbb{N}:\left|x_{k}-\ell\right| \geq \varepsilon\right\}\right)=0 .
$$

In this case, $\ell$ is called the statistical limit of the sequence $\left(x_{k}\right)$.

Definition 3. A nonempty family of sets $I \subseteq 2^{\mathbb{N}}$ is said to be an ideal on $\mathbb{N}$ if and only if

(a) $\phi \in I$,

(b) for each $A, B \in I$ one has $A \cup B \in I$,

(c) for each $B \in I$ and $A \subset B$, implies $A \in I$.

Definition 4. An ideal $I$ is an admissible ideal if $\{x\} \in I$ for each $x \in \mathbb{N}$.

Definition 5. An ideal $I \subseteq 2^{\mathbb{N}}$ is said to be nontrivial if $I \neq \phi$ and $\mathbb{N} \notin I$.

Definition 6. A nonempty family of sets $F \subseteq 2^{\mathbb{N}}$ is said to be a filter on $\mathbb{N}$ if and only if

(a) $\phi \notin F$, (b) for each $A, B \in F$ one has $A \cap B \in F$,

(c) for each $A \in F$ and $B \supset A$, implies $B \in F$.

For each ideal $I$, there is a filter $F(I)$ corresponding to $I$; that is, $F(I)=\{K \subseteq \mathbb{N}: \mathbb{N}-K \in I\}$.

Example 7. If we take $I=I_{f}=\{A \subseteq \mathbb{N}: A$ is a finite subset $\}$, then $I_{f}$ is a nontrivial admissible ideal of $\mathbb{N}$ and the corresponding convergence coincides with the usual convergence.

Example 8. If we get $I=I_{\delta}=\{A \subseteq \mathbb{N}: \delta(A)=0\}$, where $\delta(A)$ denote the asymptotic density of the set $A$, then $I_{\delta}$ is a nontrivial admissible ideal of $\mathbb{N}$ and the corresponding convergence coincides with the statistical convergence.

Definition 9. A sequence $x=\left(x_{k}\right)$ is said to be $I$-convergent to a real number $\ell$ if

$$
\left\{k \in \mathbb{N}:\left|x_{k}-\ell\right| \geq \varepsilon\right\} \in I \quad \text { for every } \varepsilon>0 .
$$

In this case, we write $I-\lim x_{k}=\ell$.

Definition 10. By a lacunary sequence $\Theta=\left(i_{j}\right), j=0,1,2, \ldots$, where $i_{0}=0$, one will mean an increasing sequence of nonnegative integers with $i_{j}-i_{j-1} \rightarrow \infty$ as $j \rightarrow \infty$, $h_{j}=i_{j}-i_{j-1}$. The intervals determined by $\Theta$ will be denoted by $\Lambda_{j}=\left(i_{j-1}, i_{j}\right]$.

Definition 11. A sequence $x=\left(x_{k}\right)$ is said to be lacunary $(L)$ statistically convergent to the number $\ell$ if, for every $\varepsilon>0$, one has

$$
\lim _{j \rightarrow \infty} \frac{1}{h_{j}}\left|\left\{k \in \Lambda_{j}:\left|x_{k}-\ell\right| \geq \varepsilon\right\}\right|=0 .
$$

The notion of lacunary ideal convergence of real sequences is introduced by Tripathy et al. [36], and Hazarika [37, 38] introduced the lacunary ideal convergent sequences of fuzzy real numbers and studied some properties.

Definition 12. Let $I \subset 2^{\mathbb{N}}$ be a nontrivial ideal. A sequence $x=\left(x_{k}\right)$ is said to be $I_{L}$-summable to a number $\ell$ if, for every $\varepsilon>0$, the set

$$
\left\{j \in \mathbb{N}: \frac{1}{h_{j}} \sum_{k \in \Lambda_{j}}\left|x_{k}-\ell\right| \geq \varepsilon\right\} \in I .
$$

Definition 13. Let $n \in \mathbb{N}$ and let $X$ be a linear space over the field $K$ of dimension $d$, where $d \geq n \geq 2$ and $K$ is the field of real or complex numbers. A real valued function $\|\cdot, \ldots, \cdot\|$ on $X^{n}$ satisfies the following four conditions:

(1) $\left\|x_{1}, x_{2}, \ldots, x_{n}\right\|=0$ if and only if $x_{1}, x_{2}, \ldots, x_{n}$ are linearly dependent in $X$;

(2) $\left\|x_{1}, x_{2}, \ldots, x_{n}\right\|$ is invariant under permutation;

(3) $\left\|\alpha x_{1}, x_{2}, \ldots, x_{n}\right\|=|\alpha|\left\|x_{1}, x_{2}, \ldots, x_{n}\right\|$ for any $\alpha \in K$;

(4) $\left\|x+x^{\prime}, x_{2}, \ldots, x_{n}\right\| \leq\left\|x, x_{2}, \ldots, x_{n}\right\|+\left\|x^{\prime}, x_{2}, \ldots, x_{n}\right\|$ is called an $n$-norm on $X$, and the pair $(X ;\|\cdot, \ldots, \cdot\|)$ is called an $n$-normed space over the field $K$. 
Definition 14. A probability distribution function is a function $F$ that is nondecreasing, left continuous on $(0, \infty)$ such that $F(0)=0$ and $F(\infty)=1$. The family of all probability distribution functions will be denoted by $\Delta^{+}$. The space $\Delta^{+}$is partially ordered by the usual pointwise ordering of functions and has both a maximal element $\varepsilon_{0}$ and a minimal element $\varepsilon_{\infty}$; these are given, respectively, by

$$
\begin{gathered}
\varepsilon_{0}(t)= \begin{cases}0, & t \leq 0, \\
1, & t>0,\end{cases} \\
\varepsilon_{\infty}(t)= \begin{cases}0, & t<\infty, \\
1, & t=\infty .\end{cases}
\end{gathered}
$$

There is a natural topology on $\Delta^{+}$that is induced by the modified Lévy metric $d_{L}[39,40]$; that is,

$$
d_{L}(F, G)=\inf \{h: \operatorname{both}[F, G ; h] \text { and }[G, F ; h] \text { hold }\}
$$

for all $F, G \in \Delta^{+}$and $h \in(0,1]$, where $[F, G, h]$ denote the condition

$$
G(t) \leq F(t+h)+h, \quad \text { for } t \in\left(0, \frac{1}{h}\right) .
$$

Convergence with respect to this metric is equivalent to weak convergence of distribution functions; that is, $\left(F_{n}\right)$ in $\Delta^{+}$ converges weakly to $F$ (written as $F_{n} \stackrel{\omega}{\rightarrow} F$ ) if and only if $F_{n}(t)$ converges to $F(t)$ at every point of continuity of the limit function $F$. Therefore, one has

$$
\begin{gathered}
F_{n} \stackrel{\omega}{\longrightarrow} F \quad \text { iff } d_{L}\left(F_{n}, F\right) \longrightarrow 0, \\
F(x)>1-x \quad \text { iff } d_{L}\left(F, \varepsilon_{0}\right)<x \text { for every } x>0 .
\end{gathered}
$$

Moreover, the metric space $\left(\Delta^{+}, d_{L}\right)$ is compact.

Definition 15. A binary operation $\star:[0,1] \times[0,1] \rightarrow[0,1]$ is said to be a continuous $t$-norm if the following conditions are satisfied:

(1) $\star$ is associative and commutative,

(2) $\star$ is continuous,

(3) $a \star 1=a$ for all $a \in[0,1]$,

(4) $a \star b \leq c \star d$ whenever $a \leq c$ and $b \leq d$ for each $a, b, c, d \in[0,1]$.

Definition 16. A binary operation $\diamond:[0,1] \times[0,1] \rightarrow[0,1]$ is said to be a continuous $t$-conorm if the following conditions are satisfied:

$(1) \diamond$ is associative and commutative,

$(2) \diamond$ is continuous,

(3) $a \diamond 0=a$ for all $a \in[0,1]$,

(4) $a \diamond b \leq c \diamond d$ whenever $a \leq c$ and $b \leq d$ for each $a, b, c, d \in[0,1]$.
Definition 17. Let $X$ be a linear space of dimension greater than one, $\star$ a continuous $t$-norm, and $\rho$ a mapping from $X^{2}$ into $D^{+}$. If the following conditions are satisfied:

(1) $\rho_{x, y}=\varepsilon_{0}$ if $x$ and $y$ are linearly dependent,

(2) $\rho_{x, y}=\rho_{y, x}$ for every $x$ and $y$ in $X$,

(3) $\rho_{\alpha x, y}(t)=\rho_{x, y}(t /|\alpha|)$ for every $t>0 ; \alpha \neq 0$ and $x ; y \epsilon$ $X$

(4) $\rho_{x+y, z}(t) \geq \rho_{x, z}(t) \star \rho_{y, z}(t)$,

then $\rho$ is called a random 2 -norm on $X$ and $(X ; \rho ; \star)$ is called a random 2-normed space.

Definition 18. Let $X$ be a linear space of dimension greater than one over a real field, $\star$ a continuous $t$-norm, and $\rho$ a mapping from $X^{n}$ into $D^{+}$. If the following conditions are satisfied:

(1) $\rho_{x_{1}, x_{2}, \ldots, x_{n}}=\varepsilon_{0} \Leftrightarrow x_{1}, x_{2}, \ldots, x_{n}$ are linearly dependent,

(2) $\rho_{x_{1}, x_{2}, \ldots, x_{n}}$ is invariant under any permutation of $x_{1}, x_{2}, \ldots, x_{n}$

(3) $\rho_{\alpha x_{1}, x_{2}, \ldots, x_{n}}(t)=\rho_{x_{1}, x_{2}, \ldots, x_{n}}(t /|\alpha|)$ for every $t>0$; $\alpha \neq 0$,

(4) $\rho_{x_{1}, x_{2}, \ldots, x_{n}+x_{n}^{\prime}}(t+s) \geq \rho_{x_{1}, x_{2}, \ldots, x_{n}}(t) \star \rho_{x_{1}, x_{2}, \ldots, x_{n}^{\prime}}(s)$,

then $\rho$ is called a random $n$-norm on $X$ and $(X ; \rho ; \star)$ is called a random $n$-normed space.

\section{Generalized Random $n$-Normed Space}

Throughout the paper let $I$ be an admissible ideal of $\mathbb{N}$. By generalizing Definition 18, we obtain a new notion of generalized random $n$-normed space as follows.

Definition 19. The five-tuple $(X, \rho, \varrho, \star, \diamond)$ is said to be generalized random $n$-normed linear space or in short GR $n$ NLS if $X$ is a linear space over the field of complex numbers $\mathbb{C}, \star$ is a continuous $t$-norm, $\diamond$ is a continuous $t$-conorm, and $\rho, \varrho$ are two mappings on $X^{n} \times(0, \infty)$ into $D^{+} \times(0, \infty)$ satisfying the following conditions for every $x=\left(x_{1}, x_{2}, \ldots, x_{n}\right) \in X^{n}$ and for each $s, t \in(0, \infty)$ :

(1) $\rho_{x_{1}, x_{2}, \ldots, x_{n}}+\varrho_{x_{1}, x_{2}, \ldots, x_{n}} \leq \varepsilon_{0}$,

(2) $\rho_{x_{1}, x_{2}, \ldots, x_{n}} \geq \varepsilon_{\infty}$,

(3) $\rho_{x_{1}, x_{2}, \ldots, x_{n}}=\varepsilon_{0}$ if and only if $x_{1}, x_{2}, \ldots, x_{n}$ are linearly dependent,

(4) $\rho_{\alpha x_{1}, x_{2}, \ldots, x_{n}}(t)=\rho_{x_{1}, x_{2}, \ldots, x_{n}}(t /|\alpha|)$ for each $\alpha \notin \mathbb{C} \backslash 0$,

(5) $\rho_{x_{1}, x_{2}, \ldots, x_{n}^{\prime}}(t) \star \rho_{x_{1}, x_{2}, \ldots, x_{n}}(s) \leq \rho_{x_{1}, x_{2}, \ldots, x_{n}^{\prime}+x_{n}}(t+s)$,

(6) $\rho_{x_{1}, x_{2}, \ldots, x_{n}}(\cdot):(0, \infty) \rightarrow[0,1]$ is continuous,

(7) $\rho_{x_{1}, x_{2}, \ldots, x_{n}}(t)$ is invariant under any permutation of $\left(x_{1}, x_{2}, \ldots, x_{n}\right)$,

(8) $\varrho_{x_{1}, x_{2}, \ldots, x_{n}}(t) \geq \varepsilon_{\infty}$,

(9) $\varrho_{x_{1}, x_{2}, \ldots, x_{n}}=\varepsilon_{\infty}$ if and only if $x_{1}, x_{2}, \ldots, x_{n}$ are linearly dependent

(10) $\varrho_{\alpha x_{1}, x_{2}, \ldots, x_{n}}(t)=\varrho_{x_{1}, x_{2}, \ldots, x_{n}}(t /|\alpha|)$ for each $\alpha \notin \mathbb{C} \backslash 0$, 
(11) $\varrho\left(x_{1}, x_{2}, \ldots, x_{n}^{\prime}, t\right) \diamond \varrho\left(x_{1}, x_{2}, \ldots, x_{n}, s\right) \quad \geq$ $\varrho\left(x_{1}, x_{2}, \ldots, x_{n}^{\prime}+x_{n}, t+s\right)$,

(12) $\varrho_{x_{1}, x_{2}, \ldots, x_{n}}(\cdot):(0, \infty) \rightarrow[0,1]$ is continuous,

(13) $\rho_{x_{1}, x_{2}, \ldots, x_{n}}(t)$ is invariant under any permutation of $\left(x_{1}, x_{2}, \ldots, x_{n}\right)$.

In this case, $(\rho, \varrho)$ is called generalized random $n$-norm on $X$ and we denote it by $(\rho, \varrho)_{n}$.

Example 20. Let $(X,\|\cdot, \ldots, \cdot\|)$ be an $n$-normed linear space. Put $a \star b=\min \{a, b\}$ and $a \diamond b=\max \{a, b\}$ for all $a, b \in[0,1]$, $\rho_{x_{1}, x_{2}, \ldots, x_{n}}(t)=t /\left(t+\left\|x_{1}, x_{2}, \ldots, x_{n}\right\|\right)$, and $\varrho_{x_{1}, x_{2}, \ldots, x_{n}}(t)=$ $\left\|x_{1}, x_{2}, \ldots, x_{n}\right\| /\left(t+\left\|x_{1}, x_{2}, \ldots, x_{n}\right\|\right)$. Then, $(X, \rho, \varrho, \star, \diamond)$ is GRnNLS.

Proof. For all $t, s \in(0, \infty)$, we have the following.

(1) Evidently, $\rho_{x_{1}, x_{2}, \ldots, x_{n}}(t)+\varrho_{x_{1}, x_{2}, \ldots, x_{n}}(t) \leq 1$.

(2) Visibly, $\rho_{x_{1}, x_{2}, \ldots, x_{n}}(t) \geq 0$.

(3) And

$$
\begin{aligned}
\rho_{x_{1}, x_{2}, \ldots, x_{n}}(t)=1 & \Longleftrightarrow \frac{t}{t+\left\|x_{1}, x_{2}, \ldots, x_{n}\right\|}=1 \\
& \Longleftrightarrow t=t+\left\|x_{1}, x_{2}, \ldots, x_{n}\right\| \\
& \Longleftrightarrow\left\|x_{1}, x_{2}, \ldots, x_{n}\right\|=0 \\
& \Longleftrightarrow x_{1}, x_{2}, \ldots, x_{n} \text { are linearly dependent. }
\end{aligned}
$$

(10)

(4) While $\left\|x_{1}, x_{2}, \ldots, x_{n}\right\|$ is invariant under any permutation of $\left(x_{1}, x_{2}, \ldots, x_{n}\right)$, then $\rho_{x_{1}, x_{2}, \ldots, x_{n}}(t)$ is invariant under any permutation of $\left(x_{1}, x_{2}, \ldots, x_{n}\right)$.

(5) Consider

$$
\begin{aligned}
\rho_{x_{1}, x_{2}, \ldots, x_{n}}\left(\frac{t}{|\alpha|}\right) & =\frac{t /|\alpha|}{t /|\alpha|+\left\|x_{1}, x_{2}, \ldots, x_{n}\right\|} \\
& =\frac{t}{t+|\alpha|\left\|x_{1}, x_{2}, \ldots, x_{n}\right\|} \\
& =\frac{t}{t+\left\|\alpha x_{1}, x_{2}, \ldots, x_{n}\right\|} \\
& =\rho_{x_{1}, x_{2}, \ldots, x_{n}}\left(\frac{t}{|\alpha|}\right) .
\end{aligned}
$$

(6) Suppose that, without loss of generality,

$$
\begin{aligned}
\rho_{x_{1}, x_{2}, \ldots, x_{n}^{\prime}}(t) \leq \rho_{x_{1}, x_{2}, \ldots, x_{n}}(s) . \\
\quad \Longrightarrow \frac{t}{t+\left\|x_{1}, x_{2}, \ldots, x_{n}^{\prime}\right\|} \leq \frac{s}{s+\left\|x_{1}, x_{2}, \ldots, x_{n}\right\|} \\
\quad \Longrightarrow t\left(s+\left\|x_{1}, x_{2}, \ldots, x_{n}\right\|\right) \\
\quad \leq s\left(t+\left\|x_{1}, x_{2}, \ldots, x_{n}^{\prime}\right\|\right) \\
\quad \Longrightarrow t\left(\left\|x_{1}, x_{2}, \ldots, x_{n}\right\|\right) \leq s\left(\left\|x_{1}, x_{2}, \ldots, x_{n}^{\prime}\right\|\right) \\
\quad \Longrightarrow\left\|x_{1}, x_{2}, \ldots, x_{n}\right\|=\frac{s}{t}\left\|x_{1}, x_{2}, \ldots, x_{n}^{\prime}\right\| .
\end{aligned}
$$

As a result,

$$
\begin{aligned}
& \left\|x_{1}, x_{2}, \ldots, x_{n}\right\|+\left\|x_{1}, x_{2}, \ldots, x_{n}^{\prime}\right\| \\
& \leq \frac{s}{t}\left\|x_{1}, x_{2}, \ldots, x_{n}^{\prime}\right\|+\left\|x_{1}, x_{2}, \ldots, x_{n}^{\prime}\right\| \\
& \quad=\frac{s+t}{t}\left\|x_{1}, x_{2}, \ldots, x_{n}^{\prime}\right\| .
\end{aligned}
$$

However,

$$
\begin{aligned}
& \left\|x_{1}, x_{2}, \ldots, x_{n}+x_{n}^{\prime}\right\| \\
& \leq\left\|x_{1}, x_{2}, \ldots, x_{n}\right\|+\left\|x_{1}, x_{2}, \ldots, x_{n}^{\prime}\right\| \\
& \leq \frac{s+t}{t}\left\|x_{1}, x_{2}, \ldots, x_{n}^{\prime}\right\|, \\
& \Longrightarrow \frac{\left\|x_{1}, x_{2}, \ldots, x_{n}+x_{n}^{\prime}\right\|}{s+t} \leq \frac{\left\|x_{1}, x_{2}, \ldots, x_{n}^{\prime}\right\|}{t} \\
& \Longrightarrow 1+\frac{\left\|x_{1}, x_{2}, \ldots, x_{n}+x_{n}^{\prime}\right\|}{s+t} \leq 1+\frac{\left\|x_{1}, x_{2}, \ldots, x_{n}^{\prime}\right\|}{t} \\
& \Longrightarrow \frac{s+t+\left\|x_{1}, x_{2}, \ldots, x_{n}+x_{n}^{\prime}\right\|}{s+t} \\
& \leq \frac{t+\left\|x_{1}, x_{2}, \ldots, x_{n}^{\prime}\right\|}{t} \\
& \Longrightarrow \frac{s+t}{s+t+\left\|x_{1}, x_{2}, \ldots, x_{n}+x_{n}^{\prime}\right\|} \\
& \geq \frac{t}{t+\left\|x_{1}, x_{2}, \ldots, x_{n}^{\prime}\right\|} \\
& \Longrightarrow \rho_{x_{1}, x_{2}, \ldots, x_{n}+x_{n}^{\prime}}(s+t) \\
& \geq \min \left\{\rho_{x_{1}, x_{2}, \ldots, x_{n}}(s), \rho_{x_{1}, x_{2}, \ldots, x_{n}^{\prime}}(t)\right\} \text {. }
\end{aligned}
$$

(7) Evidently, $\rho_{x_{1}, x_{2}, \ldots, x_{n}}(\cdot):(0, \infty) \rightarrow[0,1]$ is continuous.

(8) $\varrho_{x_{1}, x_{2}, \ldots, x_{n}}(t) \geq 0$.

(9) And

$$
\begin{aligned}
\varrho_{x_{1}, x_{2}, \ldots, x_{n}}(t)=0 & \Longleftrightarrow \frac{\left\|x_{1}, x_{2}, \ldots, x_{n}\right\|}{t+\left\|x_{1}, x_{2}, \ldots, x_{n}\right\|}=0 \\
& \Longleftrightarrow\left\|x_{1}, x_{2}, \ldots, x_{n}\right\|=0 \\
& \Longleftrightarrow x_{1}, x_{2}, \ldots, x_{n} \text { are linearly dependent. }
\end{aligned}
$$

(10) As $\left\|x_{1}, x_{2}, \ldots, x_{n}\right\|$ is invariant under any permutation of $\left(x_{1}, x_{2}, \ldots, x_{n}\right)$, then $\varrho_{x_{1}, x_{2}, \ldots, x_{n}}(t)$ is invariant under any permutation of $\left(x_{1}, x_{2}, \ldots, x_{n}\right)$. 
(11) Consider

$$
\begin{aligned}
\varrho_{\alpha x_{1}, x_{2}, \ldots, x_{n}}(t) & =\frac{\left\|\alpha x_{1}, x_{2}, \ldots, x_{n}\right\|}{t+\left\|\alpha x_{1}, x_{2}, \ldots, x_{n}\right\|} \\
& =\frac{|\alpha|\left\|x_{1}, x_{2}, \ldots, x_{n}\right\|}{t+|\alpha|\left\|x_{1}, x_{2}, \ldots, x_{n}\right\|} \\
& =\frac{\left\|x_{1}, x_{2}, \ldots, x_{n}\right\|}{t /|\alpha|+\left\|x_{1}, x_{2}, \ldots, x_{n}\right\|} \\
& =\varrho_{x_{1}, x_{2}, \ldots, x_{n}}\left(\frac{t}{|\alpha|}\right) .
\end{aligned}
$$

(12) Presume, without loss of generality, that

$$
\begin{aligned}
& \varrho_{x_{1}, x_{2}, \ldots, x_{n}}(s) \leq \rho_{x_{1}, x_{2}, \ldots, x_{n}^{\prime}}(t) . \\
& \quad \Longrightarrow \frac{\left\|x_{1}, x_{2}, \ldots, x_{n}\right\|}{s+\left\|x_{1}, x_{2}, \ldots, x_{n}\right\|} \leq \frac{\left\|x_{1}, x_{2}, \ldots, x_{n}^{\prime}\right\|}{t+\left\|x_{1}, x_{2}, \ldots, x_{n}^{\prime}\right\|} \\
& \Longrightarrow\left\|x_{1}, x_{2}, \ldots, x_{n}\right\|\left(t+\left\|x_{1}, x_{2}, \ldots, x_{n}^{\prime}\right\|\right) \\
& \leq\left\|x_{1}, x_{2}, \ldots, x_{n}^{\prime}\right\|\left(s+\left\|x_{1}, x_{2}, \ldots, x_{n}\right\|\right) \\
& \Longrightarrow t\left\|x_{1}, x_{2}, \ldots, x_{n}\right\| \leq s\left\|x_{1}, x_{2}, \ldots, x_{n}^{\prime}\right\| .
\end{aligned}
$$

Currently,

$$
\begin{aligned}
& \frac{\left\|x_{1}, x_{2}, \ldots, x_{n}+x_{n}^{\prime}\right\|}{s+t+\left\|x_{1}, x_{2}, \ldots, x_{n}+x_{n}^{\prime}\right\|}-\frac{\left\|x_{1}, x_{2}, \ldots, x_{n}^{\prime}\right\|}{t+\left\|x_{1}, x_{2}, \ldots, x_{n}^{\prime}\right\|} \\
& \leq \frac{\left\|x_{1}, x_{2}, \ldots, x_{n}\right\|+\left\|x_{1}, x_{2}, \ldots, x_{n}^{\prime}\right\|}{s+t+\left\|x_{1}, x_{2}, \ldots, x_{n}+x_{n}^{\prime}\right\|} \\
& \quad-\frac{\left\|x_{1}, x_{2}, \ldots, x_{n}^{\prime}\right\|}{t+\left\|x_{1}, x_{2}, \ldots, x_{n}^{\prime}\right\|} \\
& =\frac{t\left\|x_{1}, x_{2}, \ldots, x_{n}\right\|-s\left\|x_{1}, x_{2}, \ldots, x_{n}^{\prime}\right\|}{\left(s+t+\left\|x_{1}, x_{2}, \ldots, x_{n}+x_{n}^{\prime}\right\|\right)\left(t+\left\|x_{1}, x_{2}, \ldots, x_{n}^{\prime}\right\|\right)} .
\end{aligned}
$$

By (17),

$$
\frac{\left\|x_{1}, x_{2}, \ldots, x_{n}+x_{n}^{\prime}\right\|}{s+t+\left\|x_{1}, x_{2}, \ldots, x_{n}+x_{n}^{\prime}\right\|} \leq \frac{\left\|x_{1}, x_{2}, \ldots, x_{n}^{\prime}\right\|}{t+\left\|x_{1}, x_{2}, \ldots, x_{n}^{\prime}\right\|} \text {. }
$$

In the same way,

$$
\begin{aligned}
& \frac{\left\|x_{1}, x_{2}, \ldots, x_{n}+x_{n}^{\prime}\right\|}{s+t+\left\|x_{1}, x_{2}, \ldots, x_{n}+x_{n}^{\prime}\right\|} \\
& \quad \leq \frac{\left\|x_{1}, x_{2}, \ldots, x_{n}^{\prime}\right\|}{t+\left\|x_{1}, x_{2}, \ldots, x_{n}^{\prime}\right\|} . \\
& \quad \Longrightarrow \varrho_{x_{1}, x_{2}, \ldots, x_{n}+x_{n}^{\prime}}(s+t) \\
& \quad \leq \max \left\{\varrho_{x_{1}, x_{2}, \ldots, x_{n}}(s), \rho_{x_{1}, x_{2}, \ldots, x_{n}^{\prime}}(t)\right\} .
\end{aligned}
$$

(13) Clearly, $\rho_{x_{1}, x_{2}, \ldots, x_{n}}(\cdot):(0, \infty) \rightarrow[0,1]$ is continuous.

Remark 21. Let $(X, \rho, \varrho, \star, \diamond)$ be GRnNLS. Since $\star$ is a continuous $t$-norm and $\diamond$ is a continuous $t$-conorm, the system $(r, t)$-neighborhoods of $\theta$ (the null vector in $X$ ) with respect to $t$ is

$$
\{B(\theta, r, t): t>0,0<r<1\}
$$

where

$$
\begin{array}{r}
B(\theta, r, t)=\left\{y \in X: \rho_{y, x_{1}, x_{2}, \ldots, x_{n-1}}(t)>1-r,\right. \\
\left.\varrho_{y, x_{1}, x_{2}, \ldots, x_{n-1}}(t)<r, \text { for } t>0\right\}
\end{array}
$$

defined a first countable Hausdorff topology on $X$, called the $(\rho, \varrho)_{n}$-topology. Hence, the $(\rho, \varrho)_{n}$-topology can be completely specified by means of $(\rho, \varrho)_{n}$-convergence of sequences.

Definition 22. Let $(X, \rho, \varrho, \star, \diamond)$ be GR $n$ NLS, and let $r \in(0,1)$ and $x \in X$. The set

$$
\begin{array}{r}
B(x, r, t)=\left\{y \in X: \rho_{y-x, x_{1}, x_{2}, \ldots, x_{n-1}}(t)>1-r,\right. \\
\left.\varrho_{y-x, x_{1}, x_{2}, \ldots, x_{n-1}}(t)<r, \text { for } t>0\right\}
\end{array}
$$

is called open ball with center $x$ and radius $r$ with respect to $t$.

Definition 23. Let $(X, \rho, \varrho, *, \diamond)$ be GRnNLS. A sequence $x=$ $\left(x_{k}\right)$ in $X$ is $(\rho, \varrho)_{n}$-convergent to $\ell \in X$ with respect to the generalized random $n$-norm $(\rho, \varrho)_{n}$ if, for $r \in(0,1)$ and every $t>0$, there exists $k_{0}$ such that

$$
\begin{gathered}
\rho_{x_{k}-\ell, x_{1}, x_{2}, \ldots, x_{n-1}}(t) \geq 1-r, \\
\varrho_{x_{k}-\ell, x_{1}, x_{2}, \ldots, x_{n-1}}(t) \leq r \\
\forall k \geq k_{0} .
\end{gathered}
$$

In this case, one writes $(\rho, \varrho)_{n}-\lim x=\ell$.

Theorem 24. Let $(X,\|\cdot, \ldots, \cdot\|)$ be an n-normed linear space. Put $a \star b=\min \{a, b\}$ and $a \diamond b=\max \{a, b\}$ for all $a, b \in[0,1]$, $\rho_{x_{1}, x_{2}, \ldots, x_{n}}(t)=t /\left(t+\left\|x_{1}, x_{2}, \ldots, x_{n}\right\|\right)$, and $\varrho_{x_{1}, x_{2}, \ldots, x_{n}}(t)=$ $\left\|x_{1}, x_{2}, \ldots, x_{n}\right\| /\left(t+\left\|x_{1}, x_{2}, \ldots, x_{n}\right\|\right)$. Then, for every sequence $x=\left(x_{k}\right)$ and nonzero $x_{1}, x_{2}, \ldots, x_{n-1} \in X$, one has

$$
\lim _{k \rightarrow \infty}\left\|x_{1}, x_{2}, \ldots, x_{n-1}, x_{k}-\ell\right\|=0 \Longrightarrow(\rho, \varrho)_{n}-\lim x_{k}=\ell .
$$

Proof. Assume that $\lim _{k \rightarrow \infty}\left\|x_{1}, x_{2}, \ldots, x_{n-1}, x_{k}-\ell\right\|=0$. Then, for every $\varepsilon>0$ and for every $x_{1}, x_{2}, \ldots, x_{n-1} \in X$, there exists a positive integer $k_{0}$ such that

$$
\left\|x_{1}, x_{2}, \ldots, x_{n-1}, x_{k}-\ell\right\|<\varepsilon \text { for each } k \geq k_{0} \text {, }
$$


and, therefore, for any given $t>0$,

$$
\frac{t+\left\|x_{1}, x_{2}, \ldots, x_{n-1}, x_{k}-\ell\right\|}{t}<\frac{t+\varepsilon}{t}
$$

which is the same as

$$
\frac{t}{t+\left\|x_{1}, x_{2}, \ldots, x_{n-1}, x_{k}-\ell\right\|}>\frac{t}{t+\varepsilon}=1-\frac{\varepsilon}{t+\varepsilon} .
$$

By letting $r=\varepsilon /(t+\varepsilon) \in(0,1)$, we have

$$
\rho_{x_{k}-\ell, x_{1}, x_{2}, \ldots, x_{n-1}}(t) \geq 1-r \quad \forall k \geq k_{0} .
$$

And since $\rho_{x_{k}-\ell, x_{1}, x_{2}, \ldots, x_{n-1}}(t)=1-\varrho_{x_{k}-\ell, x_{1}, x_{2}, \ldots, x_{n-1}}(t)$, then we have

$$
\varrho_{x_{k}-\ell, x_{1}, x_{2}, \ldots, x_{n-1}}(t) \leq r \quad \forall k \geq k_{0} .
$$

This means $(\rho, \varrho)_{n}-\lim x_{k}=\ell$.

\section{4. $I_{L}^{(\rho, \varrho)_{n}}$-Cauchy and Convergence in GR $n \mathbf{N L S}$}

Remark 25. Let $(X, \rho, \varrho, \star, \diamond)$ be GRnNLS. Since $\star$ is a continuous $t$-norm and $\diamond$ is a continuous $t$-conorm, the system $(r, t)_{n}^{L}$-neighborhoods of $\theta$ with respect to $t$ are

$$
\left\{B_{L}(\theta, r, t): t>0,0<r<1\right\}
$$

where

$$
\begin{aligned}
B_{L}(\theta, r, t)= & \left\{y \in X: \frac{1}{h_{j}} \sum_{k \in \Lambda_{j}} \rho_{y_{k}, x_{1}, x_{2}, \ldots, x_{n-1}}(t)>1-r,\right. \\
& \left.\frac{1}{h_{j}} \sum_{k \in \Lambda_{j}} \varrho_{y_{k}, x_{1}, x_{2}, \ldots, x_{n-1}}(t)<r, \text { for } t>0\right\}
\end{aligned}
$$

determines a first countable Hausdorff topology on $X$, called the $(\rho, \varrho)_{n}^{L}$-topology. Thus, the $(\rho, \varrho)_{n}^{L}$-topology can be completely specified by means of $(\rho, \varrho)_{n}^{L}$-convergence of sequences.

Definition 26. Let $(X, \rho, \varrho, \star, \diamond)$ be GRnNLS, and let $r \in(0,1)$ and $x \in X$. The set

$$
\begin{aligned}
B_{L}(x, r, t)= & \left\{y \in X: \frac{1}{h_{j}} \sum_{k \in \Lambda_{j}} \rho_{y_{k}-x, x_{1}, x_{2}, \ldots, x_{n-1}}(t)>1-r,\right. \\
& \left.\frac{1}{h_{j}} \sum_{k \in \Lambda_{j}} \varrho_{y_{k}-x, x_{1}, x_{2}, \ldots, x_{n-1}}(t)<r, \text { for } t>0\right\}
\end{aligned}
$$

is called open ball with center $x$ and radius $r$ with respect to $t$.
Definition 27. Let $(X, \rho, \varrho, \star, \diamond)$ be GR $n$ NLS. A sequence $x=$ $\left(x_{k}\right)$ in $X$ is $L$-convergent to $\ell \in X$ with respect to the generalized random $n$-norm $(\rho, \varrho)_{n}$ if, for $\varepsilon \in(0,1)$ and every $t>0$, there exists $j_{0}$ such that

$$
\begin{aligned}
& \frac{1}{h_{j}} \sum_{k \in \Lambda_{j}} \rho_{x_{k}-\ell, x_{1}, x_{2}, \ldots, x_{n-1}}(t) \geq 1-\varepsilon, \\
& \frac{1}{h_{j}} \sum_{k \in \Lambda_{j}} \varrho_{x_{k}-\ell, x_{1}, x_{2}, \ldots, x_{n-1}}(t) \leq \varepsilon \\
& \forall j \geq j_{0} .
\end{aligned}
$$

In this case, one writes $(\rho, \varrho)_{n}^{L}-\lim x=\ell$.

Definition 28. Let $I \subset 2^{\mathbb{N}}$ and let $(X, \rho, \varrho, \star, \diamond)$ be GRnNLS. A sequence $x=\left(x_{k}\right)$ of elements in $X$ is said to be $I_{L}$-convergent to $\ell \in X$ with respect to the generalized random $n$-norm $(\rho, \varrho)_{n}$ if, for every $\varepsilon \in(0,1)$ and $t>0$, the set

$$
\begin{gathered}
\left\{j \in \mathbb{N}: \frac{1}{h_{j}} \sum_{k \in \Lambda_{j}} \rho_{x_{k}-\ell, x_{1}, x_{2}, \ldots, x_{n-1}}(t) \leq 1-\varepsilon\right. \text { or } \\
\left.\frac{1}{h_{j}} \sum_{k \in \Lambda_{j}} \varrho_{x_{k}-\ell, x_{1}, x_{2}, \ldots, x_{n-1}}(t) \geq \varepsilon\right\} \in I .
\end{gathered}
$$

Then, one writes $I_{L}^{(\rho, \varrho)_{n}}-\lim x=\ell$.

Example 29. Let $(\mathbb{C},\|\cdot, \ldots, \cdot\|)$ be an $n$-normed linear space; take $a \star b=a b$ and $a \diamond b=\min \{a+b, 1\}$ for all $a, b \in[0,1]$. For all $x \in \mathbb{C}$ and every $t>0$, consider

$$
\begin{aligned}
& \rho_{x_{1}, x_{2}, \ldots, x_{n}}(t)=\frac{t}{t+\left\|x_{1}, x_{2}, \ldots, x_{n}\right\|}, \\
& \varrho_{x_{1}, x_{2}, \ldots, x_{n}}(t)=\frac{\left\|x_{1}, x_{2}, \ldots, x_{n}\right\|}{t+\left\|x_{1}, x_{2}, \ldots, x_{n}\right\|} .
\end{aligned}
$$

Then, $(\mathbb{C}, \rho, \varrho, \star, \diamond)$ is GRnNLS. If we take $I=I_{\delta}$, define a sequence $x=\left(x_{k}\right)$ as follows:

$$
x_{k}= \begin{cases}1, & \text { if } k=i^{8}, \quad i \in \mathbb{N}, \\ 0, & \text { otherwise. }\end{cases}
$$

Hence, for every $\varepsilon \in(0,1)$ and $t>0$, we have

$$
\begin{array}{r}
\delta\left(\left\{j \in \mathbb{N}: \frac{1}{h_{j}} \sum_{k \in \Lambda_{j}} \rho_{x_{k}, x_{1}, x_{2}, \ldots, x_{n-1}}(t) \leq 1-\varepsilon\right.\right. \\
\left.\left.\quad \text { or } \frac{1}{h_{j}} \sum_{k \in \Lambda_{j}} \varrho_{x_{k}, x_{1}, x_{2}, \ldots, x_{n-1}}(t) \geq \varepsilon\right\}\right)=0 .
\end{array}
$$

So $I_{L}^{(\rho, \varrho)_{n}}-\lim x=0$.

Definition 30. Let $(X, \rho, \varrho, \star, \diamond)$ be GRnNLS. A sequence $x=\left(x_{k}\right)$ in $X$ is said to be a Cauchy sequence with respect 
to the generalized random $n$-norm $(\rho, \varrho)_{n}^{L}$ if, for every $t>0$ and $\varepsilon \in(0,1)$, there exists $j_{0} \in \mathbb{N}$ satisfying

$$
\begin{gathered}
\frac{1}{h_{j}} \sum_{k \in \Lambda_{j}} \rho_{x_{k}-x_{m}, x_{1}, x_{2}, \ldots, x_{n-1}}(t)>1-\varepsilon, \\
\frac{1}{h_{j}} \sum_{k \in \Lambda_{j}} \varrho_{x_{k}-x_{m}, x_{1}, x_{2}, \ldots, x_{n-1}}(t)<\varepsilon \\
\forall j, m \geq k_{0} .
\end{gathered}
$$

Definition 31. Let $(X, \rho, \varrho, *, \diamond)$ be GRnNLS. A sequence $x=$ $\left(x_{k}\right)$ in $X$ is said to be an $I_{L}$-Cauchy sequence with respect to the generalized random $n$-norm $(\rho, \varrho)_{n}^{L}$ if, for every $t>0$ and $\varepsilon \in(0,1)$, there exists $j_{0} \in \mathbb{N}$ satisfying

$$
\begin{gathered}
\left\{j \in \mathbb{N}: \frac{1}{h_{j}} \sum_{k \in \Lambda_{j}} \rho_{x_{k}-x_{m}, x_{1}, x_{2}, \ldots, x_{n-1}}(t)>1-\varepsilon,\right. \\
\left.\frac{1}{h_{j}} \sum_{k \in \Lambda_{j}} \varrho_{x_{k}-x_{m}, x_{1}, x_{2}, \ldots, x_{n-1}}(t)<\varepsilon\right\} \in F(I) .
\end{gathered}
$$

Theorem 32. Let $I \subset 2^{\mathbb{N}}$, let $(X, \rho, \varrho, \star, \diamond)$ be GRnNLS, and let $x=\left(x_{k}\right)$ be a sequence in $X$; then, for every $\varepsilon>0$ and $t>0$, one has the following:

(1) $I_{L}^{(\rho, e)_{n}}-\lim x=\ell$,

(2) $\left\{j \in \mathbb{N}:\left(1 / h_{j}\right) \sum_{k \in \Lambda_{j}} \rho_{x_{k}-\ell, x_{1}, x_{2}, \ldots, x_{n-1}}(t) \leq 1-\varepsilon\right\} \in I$ and $\left\{j \in \mathbb{N}:\left(1 / h_{j}\right) \sum_{k \in \Lambda_{j}} \varrho_{x_{k}-\ell, x_{1}, x_{2}, \ldots, x_{n-1}}(t) \geq \varepsilon\right\} \in I$,

(3) $\left\{j \in \mathbb{N}:\left(1 / h_{j}\right) \sum_{k \in \Lambda_{j}} \rho_{x_{k}-\ell, x_{1}, x_{2}, \ldots, x_{n-1}}(t) \geq 1-\varepsilon\right.$ and $\left.\left(1 / h_{j}\right) \sum_{k \in \Lambda_{j}} \varrho_{x_{k}-\ell, x_{1}, x_{2}, \ldots, x_{n-1}}(t) \leq \varepsilon\right\} \in F(I)$,

(4) $\left\{j \in \mathbb{N}:\left(1 / h_{j}\right) \sum_{k \in \Lambda_{j}} \rho_{x_{k}-\ell, x_{1}, x_{2}, \ldots, x_{n-1}}(t) \geq 1-\varepsilon\right\} \in$ $F(I)$ and $\left\{j \in \mathbb{N}:\left(1 / h_{j}\right) \sum_{k \in \Lambda_{j}} \varrho_{x_{k}-\ell, x_{1}, x_{2}, \ldots, x_{n-1}}(t) \leq\right.$ $\varepsilon\} \in F(I)$

(5) $I_{L}-\lim _{k} \rho_{x_{k}-\ell, x_{1}, x_{2}, \ldots, x_{n-1}}(t)=1$ and $I_{L}-\lim _{k} \varrho_{x_{k}-\ell, x_{1}, x_{2}, \ldots, x_{n-1}}(t)=0$.

The proof is easy, so it is omitted.

Theorem 33. Let $(X, \rho, \varrho, \star, \diamond)$ be GRnNLS and let $x=\left(x_{k}\right)$ be a sequence in $X$. If $(\rho, \varrho)_{n}^{L}-\lim x$ exists, then it is unique.

Proof. Suppose that $(\rho, \varrho)_{n}^{L}-\lim x=\ell_{1}$ and $(\rho, \varrho)_{n}^{L}-\lim x=\ell_{2}$ with $\ell_{1} \neq \ell_{2}$. Give $\varepsilon \in(0,1)$ and choose $\lambda \in(0,1)$ such that $(1-\lambda) \star(1-\lambda)>1-\varepsilon$ and $\lambda \diamond \lambda<\varepsilon$. Then, for each $t>0$, there exists $j_{1} \in \mathbb{N}$ such that

$$
\begin{aligned}
\frac{1}{h_{j}} \sum_{k \in \Lambda_{j}} \rho_{x_{k}-\ell_{1}, x_{1}, x_{2}, \ldots, x_{n-1}}(t) & >1-\varepsilon, \\
\frac{1}{h_{j}} \sum_{k \in \Lambda_{j}} \varrho_{x_{k}-\ell_{1}, x_{1}, x_{2}, \ldots, x_{n-1}}(t) & <\varepsilon \\
\forall j & \geq j_{1} .
\end{aligned}
$$

Also, there exists $j_{2} \in \mathbb{N}$ such that

$$
\begin{aligned}
\frac{1}{h_{j}} \sum_{k \in \Lambda_{j}} \rho_{x_{k}-\ell_{2}, x_{1}, x_{2}, \ldots, x_{n-1}}(t) & >1-\varepsilon, \\
\frac{1}{h_{j}} \sum_{k \in \Lambda_{j}} \varrho_{x_{k}-\ell_{2}, x_{1}, x_{2}, \ldots, x_{n-1}}(t) & <\varepsilon \\
\forall j & \geq j_{2} .
\end{aligned}
$$

Now, consider $j_{0}=\max \left\{j_{1}, j_{2}\right\}$. Then, for $j \geq j_{0}$, we find a $s \in \mathbb{N}$ such that

$$
\begin{aligned}
& \rho_{x_{s}-\ell_{1}, x_{1}, x_{2}, \ldots, x_{n-1}}\left(\frac{t}{2}\right)>\frac{1}{h_{j}} \sum_{k \in \Lambda_{j}} \rho_{x_{k}-\ell_{1}, x_{1}, x_{2}, \ldots, x_{n-1}}\left(\frac{t}{2}\right) \geq 1-\lambda, \\
& \rho_{x_{s}-\ell_{2}, x_{1}, x_{2}, \ldots, x_{n-1}}\left(\frac{t}{2}\right)>\frac{1}{h_{j}} \sum_{k \in \Lambda_{j}} \rho_{x_{k}-\ell_{2}, x_{1}, x_{2}, \ldots, x_{n-1}}\left(\frac{t}{2}\right) \geq 1-\lambda .
\end{aligned}
$$

Then, we get

$$
\begin{aligned}
\rho_{\ell_{1}-\ell_{2}, x_{1}, x_{2}, \ldots, x_{n-1}}(t) \geq & \rho_{x_{s}-\ell_{1}, x_{1}, x_{2}, \ldots, x_{n-1}}\left(\frac{t}{2}\right) \\
& \star \rho_{x_{s}-\ell_{2}, x_{1}, x_{2}, \ldots, x_{n-1}}\left(\frac{t}{2}\right) \\
> & (1-\lambda) \star(1-\lambda)>1-\varepsilon .
\end{aligned}
$$

Since $\varepsilon>0$ is arbitrary, we have $\rho_{\ell_{1}-\ell_{2}, x_{1}, x_{2}, \ldots, x_{n-1}}(t)=1$ for all $t>0$. By using a similar technique, it can be proved that $\varrho_{\ell_{1}-\ell_{2}, x_{1}, x_{2}, \ldots, x_{n-1}}(t)=0$ for all $t>0$; hence, $\ell_{1}=\ell_{2}$.

Theorem 34. Let $(X, \rho, \varrho, \star, \diamond)$ be GRnNLS and let $x=\left(x_{k}\right)$ be a sequence in $X$. Then, one has

$$
(\rho, \varrho)_{n}^{L}-\lim x=\ell \Longrightarrow I_{L}^{(\rho, \rho)_{n}}-\lim x=\ell .
$$

Proof. Let $(\rho, \varrho)_{n}^{L}-\lim x=\ell$, and, then, for all $t>0$ and given $\varepsilon \in(0,1)$, there exists $j_{0} \in \mathbb{N}$ such that

$$
\begin{aligned}
\frac{1}{h_{j}} \sum_{k \in \Lambda_{j}} \rho_{x_{k}-\ell, x_{1}, x_{2}, \ldots, x_{n-1}}(t) & >1-\varepsilon, \\
\frac{1}{h_{j}} \sum_{k \in \Lambda_{j}} \varrho_{x_{k}-\ell, x_{1}, x_{2}, \ldots, x_{n-1}}(t) & <\varepsilon \\
\forall j & \geq j_{0} .
\end{aligned}
$$

Since $I$ is an admissible ideal and

$$
\begin{aligned}
G= & \left\{j \in \mathbb{N}: \frac{1}{h_{j}} \sum_{k \in \Lambda_{j}} \rho_{x_{k}-\ell, x_{1}, x_{2}, \ldots, x_{n-1}}(t) \leq 1-\varepsilon\right. \text { or } \\
& \left.\frac{1}{h_{j}} \sum_{k \in \Lambda_{j}} \varrho_{x_{k}-\ell, x_{1}, x_{2}, \ldots, x_{n-1}}(t) \geq \varepsilon\right\} \\
\subseteq & \left.\subseteq 1,2,3, \ldots, j_{0}-1\right\},
\end{aligned}
$$

we get $G \in I$. So $I_{L}^{(\rho, \varrho)_{n}}-\lim x=\ell$. 
Theorem 35. Let $(X, \rho, \varrho, \star, \diamond)$ be GRnNLS and let $x=\left(x_{k}\right)$ be a sequence in $X$. If $I_{L}^{(\rho, \varrho)_{n}}-\lim x$ exists, then it is unique.

The proof follows by using Theorems 33 and 34 .

Theorem 36. Let $(X, \rho, \varrho, \star, \diamond)$ be GRnNLS and let $x=\left(x_{k}\right)$ be a sequence in $X$. If $(\rho, \varrho)_{n}^{L}-\lim x=\ell$, then there exists a subsequence $\left(x_{m_{k}}\right)$ of $x=\left(x_{k}\right)$ such that $(\rho, \varrho)_{n}-\lim x_{m_{k}}=\ell$.

Proof. Let $(\rho, \varrho)_{n}^{L}-\lim x=\ell$. Then, for all $t>0$ and given $\varepsilon \in(0,1)$, there exists $j_{0} \in \mathbb{N}$ such that

$$
\begin{aligned}
\frac{1}{h_{j}} \sum_{k \in \Lambda_{j}} \rho_{x_{k}-\ell, x_{1}, x_{2}, \ldots, x_{n-1}}(t)>1-\varepsilon, \\
\frac{1}{h_{j}} \sum_{k \in \Lambda_{j}} \varrho_{x_{k}-\ell, x_{1}, x_{2}, \ldots, x_{n-1}}(t)<\varepsilon \\
\forall j \geq j_{0} .
\end{aligned}
$$

Observably, for each $j \geq j_{0}$, we can take an $m_{k} \in \Lambda_{j}$ such that

$$
\begin{gathered}
\rho_{x_{m_{k}}-L, x_{1}, x_{2}, \ldots, x_{n-1}}(t)>\frac{1}{h_{j}} \sum_{k \in \Lambda} \rho_{x_{k}-\ell_{1}, x_{1}, x_{2}, \ldots, x_{n-1}}(t)>1-\varepsilon, \\
\varrho_{x_{m_{k}}-\ell, x_{1}, x_{2}, \ldots, x_{n-1}}(t)<\frac{1}{h_{j}} \sum_{k \in \Lambda_{j}} \rho_{x_{k}-\ell, x_{1}, x_{2}, \ldots, x_{n-1}}(t)<\varepsilon .
\end{gathered}
$$

It follows that $(\rho, \varrho)_{n}-\lim x_{m_{k}}=\ell$.

We create the following two results without proofs, since they can be easily recognized.

Theorem 37. Let $(X, \rho, \varrho, \star, \diamond)$ be GRnNLS. If a sequence $x=$ $\left(x_{k}\right)$ in $X$ is Cauchy sequence with respect to the generalized random $n$-norm $(\rho, \varrho)_{n}^{L}$, then it is $I_{L}$-Cauchy sequence with respect to the same norm.

Theorem 38. Let $(X, \rho, \varrho, \star, \diamond)$ be GRnNLS. If a sequence $x=$ $\left(x_{k}\right)$ in $X$ is Cauchy sequence with respect to the generalized random n-norm $(\rho, \varrho)_{n}^{L}$, then there is a subsequence of $x=\left(x_{k}\right)$ which is ordinary Cauchy sequence with respect to the norm $(\rho, \varrho)_{n}$.

\section{5. $I_{L}$-Limit Point, $I_{L}$-Cluster Point, and $I_{L}$-Cauchy Sequence in GR $n \mathbf{N L S}$}

Definition 39. Let $(X, \rho, \varrho, \star, \diamond)$ be GRnNLS, and if a sequence $x=\left(x_{k}\right)$ in $X$, then one has the following.

(1) An element $\ell \in X$ is said to be $I_{L}$-limit point of $x=$ $\left(x_{k}\right)$ if there is a set

$$
\begin{gathered}
\mathbb{M}=\left\{m_{1}<m_{2}<\cdots<m_{k}<\cdots\right\} \subset \mathbb{N} \\
\text { with } \mathbb{M}^{\prime}=\left\{j \in \mathbb{N}: m_{k} \in \Lambda_{j}\right\} \in F(I), \\
(\rho, \varrho)_{n}^{L}-\lim x_{m_{k}}=\ell .
\end{gathered}
$$

(2) An element $\ell \in X$ is said to be $I_{L}$-cluster point of $x=$ $\left(x_{k}\right)$ if, for every $t>0$ and $\varepsilon \in(0,1)$, one has

$$
\begin{array}{r}
\left\{j \in \mathbb{N}: \frac{1}{h_{j}} \sum_{k \in \Lambda} \rho_{x_{k}-\ell, x_{1}, x_{2}, \ldots, x_{n-1}}(t)>1-\varepsilon,\right. \\
\left.\frac{1}{h_{j}} \sum_{k \in \Lambda} \varrho_{x_{k}-\ell, x_{1}, x_{2}, \ldots, x_{n-1}}(t)<\varepsilon\right\} \in F(I) .
\end{array}
$$

By $\bigwedge_{(\rho, \rho)_{n}^{L}}(x)$ we denote the set of all $I_{L}$-limit points and $V_{(\rho, \varrho)_{n}^{L}}(x)$ the set of all $I_{L}$-cluster points in $X$, respectively.

Definition 40. Let $(X, \rho, \varrho, \star, \diamond)$ be GR $n$ NLS. A sequence $x=$ $\left(x_{k}\right)$ in $X$ is said to be $I_{L}^{*}$-Cauchy sequence with respect to the generalized random $n$-norm $(\rho, \varrho)_{n}^{L}$ if

(i) there exists a set $\mathbb{M}=\left\{m_{1}<m_{2}<\cdots<m_{k}, \ldots\right\} \subset \mathbb{N}$ such that $\mathbb{M}^{\prime}=\left\{j \in \mathbb{N}: m_{k} \in \Lambda_{j}\right\} \in F(I)$;

(ii) the subsequence $\left(x_{m_{k}}\right)$ of $x=\left(x_{k}\right)$ is a Cauchy sequence with respect to the generalized random $n$ $\operatorname{norm}(\rho, \varrho)_{n}^{L}$.

Theorem 41. Let $(X, \rho, \varrho, \star, \diamond)$ be GRnNLS. For each sequence $x=\left(x_{k}\right)$ in $X$, one has

$$
\bigwedge_{(\rho, \varrho)_{n}^{L}}(x) \subset \bigvee_{(\rho, \varrho)_{n}^{L}}(x) .
$$

Proof. Let $\ell \in \bigwedge_{(\rho, \varrho)_{n}^{L}}(x)$; then there exists a set $\mathbb{M} \subset \mathbb{N}$ such that $\mathbb{M}^{\prime} \in F(I)$, where $\mathbb{M}$ and $\mathbb{M}^{\prime}$ are as in Definition 39, satisfies $(\rho, \varrho)_{n}^{L}-\lim x_{m_{k}}=\ell$. Thus, for every $t>0$ and $\varepsilon \in(0,1)$, there exists $j_{0} \in \mathbb{N}$ such that

$$
\begin{aligned}
\frac{1}{h_{j}} \sum_{k \in \Lambda_{j}} \rho_{x_{m_{k}}-\ell, x_{1}, x_{2}, \ldots, x_{n-1}}(t) & >1-\varepsilon, \\
\frac{1}{h_{j}} \sum_{k \in \Lambda_{j}} \varrho_{x_{m_{k}}-\ell, x_{1}, x_{2}, \ldots, x_{n-1}}(t) & <\varepsilon \\
\forall j & \geq j_{0} .
\end{aligned}
$$

Thus, we have

$$
\begin{aligned}
& G=\{j \in \mathbb{N}: \frac{1}{h_{j}} \sum_{k \in \Lambda} \rho_{x_{m_{k}}-\ell, x_{1}, x_{2}, \ldots, x_{n-1}}(t)>1-\varepsilon, \\
&\left.\frac{1}{h_{j}} \sum_{k \in \Lambda} \rho_{x_{m_{k}}-\ell, x_{1}, x_{2}, \ldots, x_{n-1}}(t)<\varepsilon\right\} \\
& \supseteq \mathbb{M}^{\prime} \backslash\left\{m_{1}, m_{2}, \ldots, m_{j_{0}}\right\} .
\end{aligned}
$$

Since $I$ is an admissible ideal, we have

$$
\begin{aligned}
& \mathbb{M}^{\prime} \backslash\left\{m_{1}, m_{2}, \ldots, m_{j_{0}}\right\} \in F(I) \\
& \text { and so } G \in F(I) \text {. Hence } \ell \in \bigvee_{(\rho, \varrho)_{n}^{L}}(x) .
\end{aligned}
$$


Theorem 42. Let $(X, \rho, \varrho, \star, \diamond)$ be GRnNLS. For each sequence $x=\left(x_{k}\right)$ in $X$, the set $\bigwedge_{(\rho, \rho)_{n}^{L}}(x)$ is closed set in $X$ with respect to the usual topology induced by the generalized random n-norm $(\rho, \varrho)_{n}^{L}$.

Proof. Let $y \in \overline{\bigwedge_{(\rho, \varrho)_{n}^{L}}(x)}$. Take $t>0$ and $\varepsilon \in(0,1)$. Then, there exists $\ell_{0} \in \bigwedge_{(\rho, \rho)_{n}^{L}}^{L}(x) \cap B_{L}(y, \varepsilon, t)$. Choose $\delta>0$ such that $B_{L}\left(\ell_{0}, \delta, t\right) \subset B_{L}(y, \varepsilon, t)$. We have

$$
\begin{aligned}
& G=\left\{j \in \mathbb{N}: \frac{1}{h_{j}} \sum_{k \in \Lambda_{j}} \rho_{x_{k}-y, x_{1}, x_{2}, \ldots, x_{n-1}}(t)>1-\varepsilon,\right. \\
& \left.\frac{1}{h_{j}} \sum_{k \in \Lambda_{j}} \varrho_{x_{k}-y, x_{1}, x_{2}, \ldots, x_{n-1}}(t)<\varepsilon\right\} \\
& \supseteq\left\{j \in \mathbb{N}: \frac{1}{h_{j}} \sum_{k \in \Lambda_{j}} \rho_{x_{k}-\ell_{0}, x_{1}, x_{2}, \ldots, x_{n-1}}(t)>1-\delta,\right. \\
& \left.\frac{1}{h_{j}} \sum_{k \in \Lambda_{j}} \varrho_{x_{k}-\ell_{0}, x_{1}, x_{2}, \ldots, x_{n-1}}(t)<\delta\right\}=H .
\end{aligned}
$$

Thus, $H \in F(I)$ and so $G \in F(I)$. Hence, $y \in \bigwedge_{(\rho, \varrho)_{n}^{L}}(x)$.

Theorem 43. Let $(X, \rho, \varrho, \star, \diamond)$ be GRnNLS and $x=\left(x_{k}\right)$ in $X$. Then, the following statements are equivalent:

(1) $\ell$ is a $I_{L}$-limit point of $x$;

(2) there exist two sequences $y$ and $z$ in $X$ such that

$$
\begin{gathered}
x=y+z, \quad(\rho, \varrho)_{n}^{L}-\lim y=\ell, \\
\left\{j \in \mathbb{N}: k \in \Lambda_{j}, z_{k} \neq \theta\right\} \in I,
\end{gathered}
$$

where $\theta$ is the zero element in $X$.

Proof. Let (1) hold; then, there exist sets $\mathbb{M}$ and $\mathbb{M}^{\prime}$ as in Definition 39 such that

$$
\mathbb{M}^{\prime} \notin I, \quad(\rho, \varrho)_{n}^{L}-\lim x_{m_{k}}=\ell .
$$

Define the sequences $y$ and $z$ as follows:

$$
\begin{gathered}
y_{k}= \begin{cases}x_{k}, & \text { if } k \in \Lambda_{j} ; j \in \mathbb{M}^{\prime}, \\
\ell, & \text { otherwise, }\end{cases} \\
z_{k}= \begin{cases}\theta, & \text { if } k \in \Lambda_{j} ; j \in \mathbb{M}^{\prime}, \\
x_{k}-\ell, & \text { otherwise. }\end{cases}
\end{gathered}
$$

Consider the case $k \in \Lambda_{j}$ such that $j \in \mathbb{N}-\mathbb{M}^{\prime}$. Then, for each $\varepsilon \in(0,1)$ and $t>0$, we get

$$
\begin{gathered}
\rho_{y_{k}-\ell, x_{1}, x_{2}, \ldots, x_{n-1}}(t)=1>1-\varepsilon, \\
\varrho_{y_{k}-\ell, x_{1}, x_{2}, \ldots, x_{n-1}}(t)=0<\varepsilon .
\end{gathered}
$$

Thus, in this case,

$$
\begin{gathered}
\frac{1}{h_{j}} \sum_{k \in \Lambda_{j}} \rho_{y_{k}-\ell, x_{1}, x_{2}, \ldots, x_{n-1}}(t)=1>1-\varepsilon, \\
\frac{1}{h_{j}} \sum_{k \in \Lambda_{j}} \varrho_{y_{k}-\ell, x_{1}, x_{2}, \ldots, x_{n-1}}(t)=0<\varepsilon .
\end{gathered}
$$

For that, $(\rho, \varrho)_{n}^{L}-\lim y=\ell$. Now, we have

$$
\begin{aligned}
& \left\{j \in \mathbb{N}: k \in \Lambda_{j}, Z_{k} \neq \theta\right\} \subset \mathbb{N}-\mathbb{M}^{\prime} \\
& \text { and so }\left\{j \in \mathbb{N}: k \in \Lambda_{j}, Z_{k} \neq \theta\right\} \in I .
\end{aligned}
$$

Now, suppose that (2) holds. Let $\mathbb{M}^{\prime}=\left\{j \in \mathbb{N}: k \in \Lambda_{j}, Z_{k}=\right.$ $\theta$. Then, obviously $\mathbb{M}^{\prime} \in F(I)$ and so it is an infinite set. Form the set

$$
\begin{aligned}
& \mathbb{M}=\left\{m_{1}<m_{2}<\cdots<m_{k}<\cdots\right\} \subset \mathbb{N} \\
& \text { such that } m_{k} \in \Lambda_{j}, z_{m_{k}}=\theta
\end{aligned}
$$

Since $x_{m_{k}}=y_{m_{k}}$ and $(\rho, \varrho)_{n}^{L}-\lim y=\ell$, we find that $(\rho, \varrho)_{n}^{L}-$ $\lim x_{m_{k}}=\ell$. This completes the proof.

Theorem 44. Let $(X, \rho, \varrho, \star, \diamond)$ be GRnNLS and let $x=\left(x_{k}\right)$ be a sequence in $X$. Let I be a nontrivial ideal. If there is a $I_{L}^{(\rho, \varrho)_{n}}$-convergent sequence $y=\left(y_{k}\right)$ in $X$ such that $\{k \in \mathbb{N}$ : $\left.y_{k} \neq x_{k}\right\} \in I$, then $x$ is also $I_{L}^{(\rho, \varrho)_{n}}$-convergent.

Proof. Suppose that $\left\{k \in \mathbb{N}: y_{k} \neq x_{k}\right\} \in I$ and $I_{L}^{(\rho, \varrho)_{n}}-\lim y=$ $\ell$. Then, for every $\varepsilon \in(0,1)$ and $t>0$, the set

$$
\begin{gathered}
\left\{j \in \mathbb{N}: \frac{1}{h_{j}} \sum_{k \in \Lambda_{j}} \rho_{y_{k}-\ell, x_{1}, x_{2}, \ldots, x_{n-1}}(t) \leq 1-\varepsilon\right. \text { or } \\
\left.\frac{1}{h_{j}} \sum_{k \in \Lambda_{j}} \varrho_{y_{k}-\ell, x_{1}, x_{2}, \ldots, x_{n-1}}(t) \geq \varepsilon\right\} \in I .
\end{gathered}
$$

For every $0<\varepsilon<1$ and $t>0$, we have

$$
\begin{aligned}
& \left\{j \in \mathbb{N}: \frac{1}{h_{j}} \sum_{k \in \Lambda_{j}} \rho_{x_{k}-\ell, x_{1}, x_{2}, \ldots, x_{n-1}}(t) \leq 1-\varepsilon\right. \text { or } \\
& \left.\frac{1}{h_{j}} \sum_{k \in \Lambda_{j}} \varrho_{x_{k}-\ell, x_{1}, x_{2}, \ldots, x_{n-1}}(t) \geq \varepsilon\right\} \\
& \subseteq\left\{k \in \mathbb{N}: y_{k} \neq x_{k}\right\} \\
& \cup\left\{j \in \mathbb{N}: \frac{1}{h_{j}} \sum_{k \in \Lambda} \rho_{y_{k}-\ell, x_{1}, x_{2}, \ldots, x_{n-1}}(t) \leq 1-\varepsilon \quad\right. \text { or } \\
& \left.\frac{1}{h_{j}} \sum_{k \in \Lambda} \varrho_{y_{k}-\ell, x_{1}, x_{2}, \ldots, x_{n-1}}(t) \geq \varepsilon\right\} .
\end{aligned}
$$


As both of the sets of the right-hand side are in $I$, we have that

$$
\begin{gathered}
\left\{j \in \mathbb{N}: \frac{1}{h_{j}} \sum_{k \in \Lambda_{j}} \rho_{x_{k}-\ell, x_{1}, x_{2}, \ldots, x_{n-1}}(t) \leq 1-\varepsilon\right. \text { or } \\
\left.\frac{1}{h_{j}} \sum_{k \in \Lambda_{j}} \varrho_{x_{k}-\ell, x_{1}, x_{2}, \ldots, x_{n-1}}(t) \geq \varepsilon\right\} \in I .
\end{gathered}
$$

And the proof of the theorem follows.

The proof of the following result can be easily reputable from the definitions.

Theorem 45. Let $(X, \rho, \varrho, \star, \diamond)$ be GRnNLS. If a sequence $x=$ $\left(x_{k}\right)$ in $X$ is $I_{L}^{*}$-Cauchy sequence with respect to the generalized random n-norm $(\rho, \varrho)_{n}^{L}$, then it is $I_{L}$-Cauchy sequence also.

\section{Conflict of Interests}

The authors declare that there is no conflict of interests regarding the publication of this paper.

\section{Acknowledgments}

Many thanks are due to the editor and the anonymous referee for careful reading of the paper and valuable suggestions which helped in improving an earlier version of it.

\section{References}

[1] H. Fast, "Sur la convergence statistique," Colloquium Mathematicum, vol. 2, pp. 241-244, 1951.

[2] H. Steinhaus, "Sur la convergence ordinaire et la convergence asymptotique," Colloquium Mathematicum, vol. 2, pp. 73-74, 1951.

[3] J. A. Fridy, "On statistical convergence," Analysis, vol. 5, no. 4, pp. 301-313, 1985.

[4] S. A. Mohiuddine and M. Aiyub, "Lacunary statistical convergence in random 2-normed spaces," Applied Mathematics \& Information Sciences, vol. 6, no. 3, pp. 581-585, 2012.

[5] T. Šalát, "On statistically convergent sequences of real numbers," Mathematica Slovaca, vol. 30, no. 2, pp. 139-150, 1980.

[6] I. J. Maddox, "Statistical convergence in a locally convex space," Mathematical Proceedings of the Cambridge Philosophical Society, vol. 104, no. 1, pp. 141-145, 1988.

[7] P. Kostyrko, T. Šalát, and W. Wilczyński, "I-convergenceconvergence," Real Analysis Exchange, vol. 26, no. 2, pp. 669$685,2000 / 01$.

[8] K. Dems, "On I-Cauchy sequences," Real Analysis Exchange, vol. 30, no. 1, pp. 123-128, 2004-2005.

[9] M. Mursaleen, S. A. Mohiuddine, and O. H. H. Edely, "On the ideal convergence of double sequences in intuitionistic fuzzy normed spaces," Computers \& Mathematics with Applications, vol. 59, no. 2, pp. 603-611, 2010.

[10] H. Cakalli and B. Hazarika, "Ideal quasi-Cauchy sequences," Journal of Inequalities and Applications, vol. 2012, article 234, 2012.

[11] P. Das, P. Kostyrko, W. Wilczyński, and P. Malik, "I and $I^{*}$ convergence of double sequences," Mathematica Slovaca, vol. 58 , no. 5, pp. 605-620, 2008.
[12] K. Menger, "Statistical metrics," Proceedings of the National Academy of Sciences of the United States of America, vol. 28, pp. 535-537, 1942.

[13] A. N. Šerstnev, Random Normed Spaces: Problems of Completeness, vol. 122, 1962.

[14] A. N. Šerstnev, "On the concept of a stochastic normalized space," Doklady Akademii Nauk SSSR, vol. 149, pp. 280-283, 1963.

[15] C. Alsina, B. Schweizer, and A. Sklar, "On the definition of a probabilistic normed space," Aequationes Mathematicae, vol. 46, no. 1-2, pp. 91-98, 1993.

[16] M. Mursaleen and S. A. Mohiuddine, "On ideal convergence in probabilistic normed spaces," Mathematica Slovaca, vol. 62, no. 1, pp. 49-62, 2012.

[17] M. Mursaleen and A. Alotaibi, "On I-convergence in random 2-normed spaces," Mathematica Slovaca, vol. 61, no. 6, pp. 933940, 2011.

[18] M. Gürdal and I. Açık, "On I-Cauchy sequences in 2-normed spaces," Mathematical Inequalities \& Applications, vol. 11, no. 2, pp. 349-354, 2008.

[19] U. Yamanc1 and M. Gürdal, "On lacunary ideal convergence in random 2-normed space," Journal of Mathematics, vol. 2013, Article ID 868457, 8 pages, 2013.

[20] B. Hazarika, "On generalized difference ideal convergence in random 2-normed spaces," Filomat, vol. 26, no. 6, pp. 1273-1282, 2012.

[21] V. Kumar and B. Lafuerza-Guillén, "On ideal convergence of double sequences in probabilistic normed spaces," Acta Mathematica Sinica, vol. 28, no. 8, pp. 1689-1700, 2012.

[22] K. Kumar and V. Kumar, "On the $I$ and $I^{*}$-Cauchy sequences in probabilistic normed spaces," Mathematical Sciences, vol. 2, no. 1, pp. 47-58, 2008.

[23] B. K. Lahiri and P. Das, " $I$ and $I^{*}$-convergence in topological spaces," Mathematica Bohemica, vol. 130, no. 2, pp. 153-160, 2005.

[24] S. A. Mohiuddine, H. Şevli, and M. Cancan, "Statistical convergence in fuzzy 2-normed space," Journal of Computational Analysis and Applications, vol. 12, no. 4, pp. 787-798, 2010.

[25] A. Şahiner, M. Gürdal, S. Saltan, and H. Gunawan, "Ideal convergence in 2-normed spaces," Taiwanese Journal of Mathematics, vol. 11, no. 5, pp. 1477-1484, 2007.

[26] B. Schweizer and A. Sklar, "Statistical metric spaces," Pacific Journal of Mathematics, vol. 10, pp. 313-334, 1960.

[27] S. Gähler, "Lineare 2-normierte Räume," Mathematische Nachrichten, vol. 28, pp. 1-43, 1964.

[28] S. Gähler, "Untersuchungen uber verallgemeinerte mmetrische Raume, I, II, III," Mathematische Nachrichten, vol. 40, pp. 165-189, 1969.

[29] A. A. Bakery, E. A. Elnour Mohamed, and M. Alamin Ahmed, "Some generalized difference sequence spaces defined by ideal convergence and Musielak-Orlicz function," Abstract and Applied Analysis, vol. 2013, Article ID 972363, 9 pages, 2013.

[30] A. A. Bakery, "Generalized difference $\gamma$-sequence spaces defined by ideal convergence and the Musielak-Orlicz function," Abstract and Applied Analysis, vol. 2013, Article ID 123798, 14 pages, 2013.

[31] J. A. Fridy and C. Orhan, "Lacunary statistical convergence," Pacific Journal of Mathematics, vol. 160, no. 1, pp. 43-51, 1993.

[32] M. Mursaleen and S. A. Mohiuddine, "On lacunary statistical convergence with respect to the intuitionistic fuzzy normed 
space," Journal of Computational and Applied Mathematics, vol. 233, no. 2, pp. 142-149, 2009.

[33] P. Debnath, "Lacunary ideal convergence in intuitionistic fuzzy normed linear spaces," Computers \& Mathematics with Applications, vol. 63, no. 3, pp. 708-715, 2012.

[34] S. A. Mohiuddine and E. Savaş, "Lacunary statistically convergent double sequences in probabilistic normed spaces," Annali dell'Universitá di Ferrara, vol. 58, no. 2, pp. 331-339, 2012.

[35] I. H. Jebril and H. Dutta, "Generalization of $n$-Normed space," General Mathematics Notes, vol. 1, no. 1, pp. 8-19, 2010.

[36] B. C. Tripathy, B. Hazarika, and B. Choudhary, "Lacunary Iconvergent sequences," Kyungpook Mathematical Journal, vol. 52, no. 4, pp. 473-482, 2012.

[37] B. Hazarika, "Lacunary I-convergent sequence of fuzzy real numbers," The Pacific Journal of Science and Technology, vol. 10, no. 2, pp. 203-206, 2009.

[38] B. Hazarika, "Fuzzy real valued lacunary I-convergent sequences," Applied Mathematics Letters, vol. 25, no. 3, pp. 466-470, 2012.

[39] B. Schweizer and A. Sklar, Probabilistic Metric Spaces, NorthHolland Series in Probability and Applied Mathematics, NorthHolland, New York, NY, USA, 1983.

[40] D. A. Sibley, "A metric for weak convergence of distribution functions," The Rocky Mountain Journal of Mathematics, vol. 1, no. 3, pp. 427-430, 1971. 


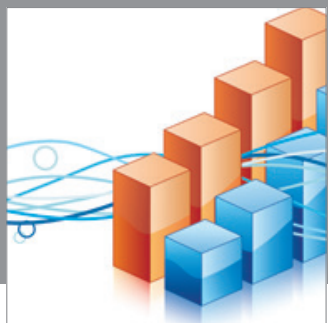

Advances in

Operations Research

mansans

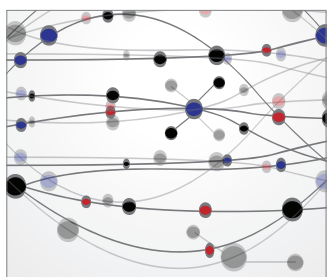

The Scientific World Journal
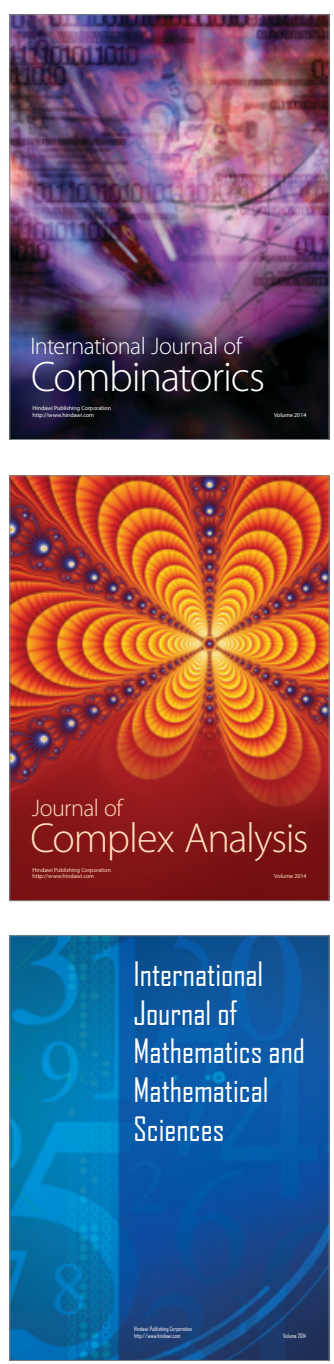
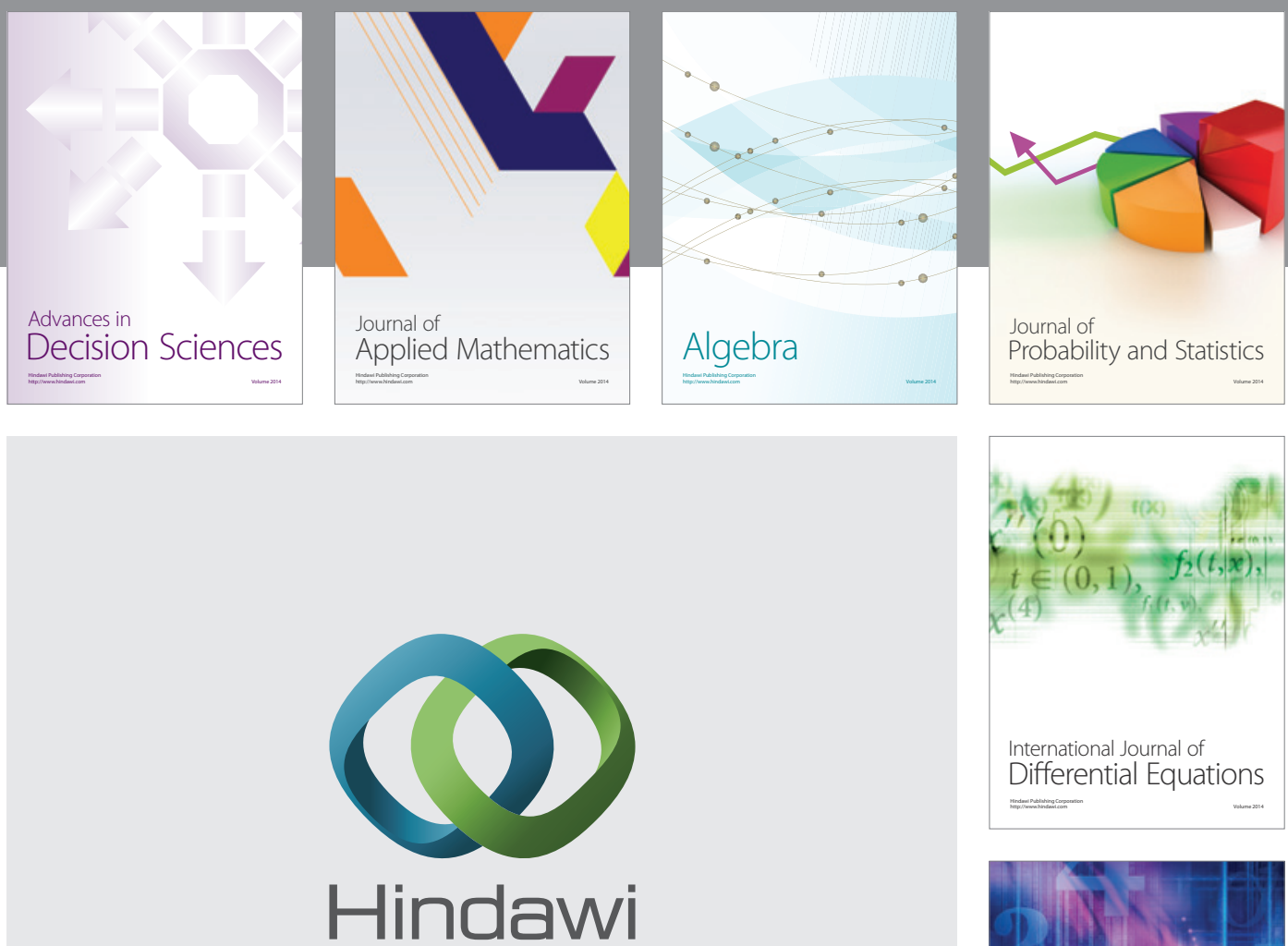

Submit your manuscripts at http://www.hindawi.com
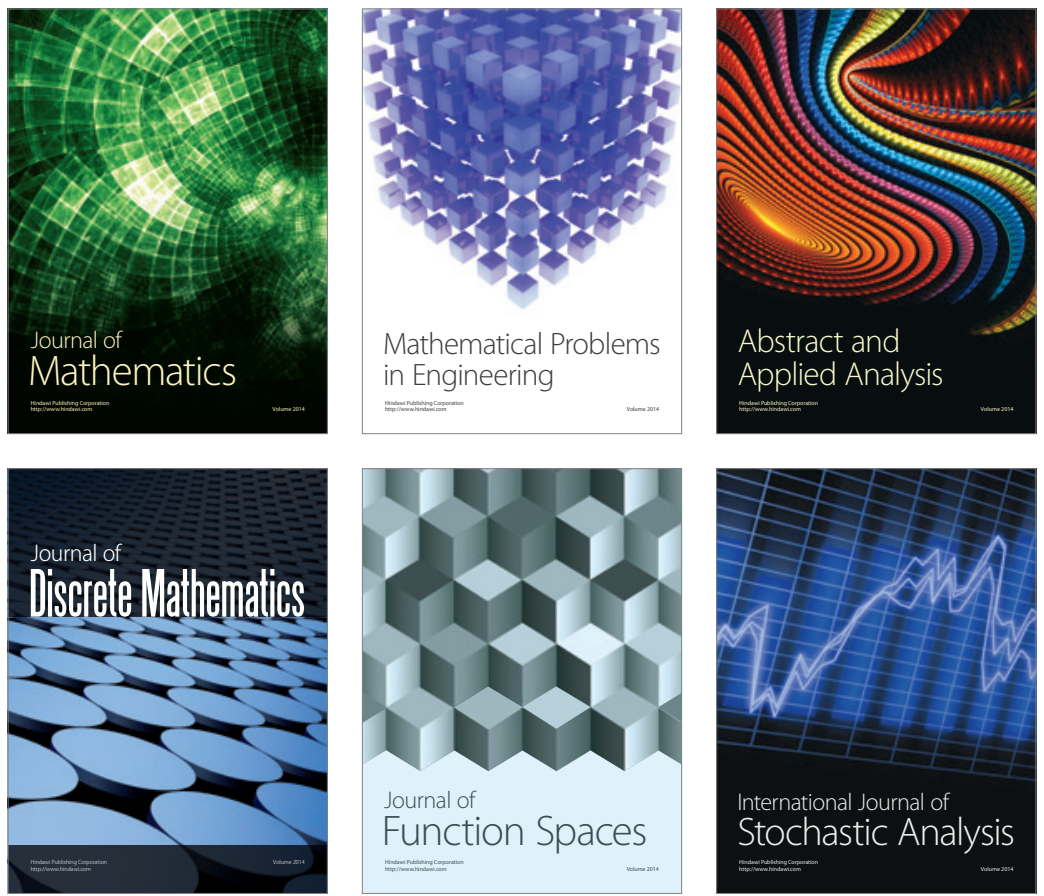

Journal of

Function Spaces

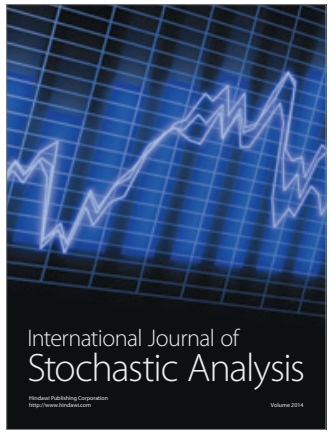

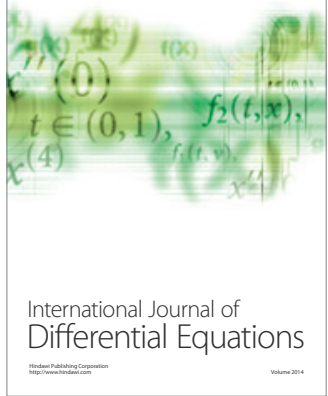
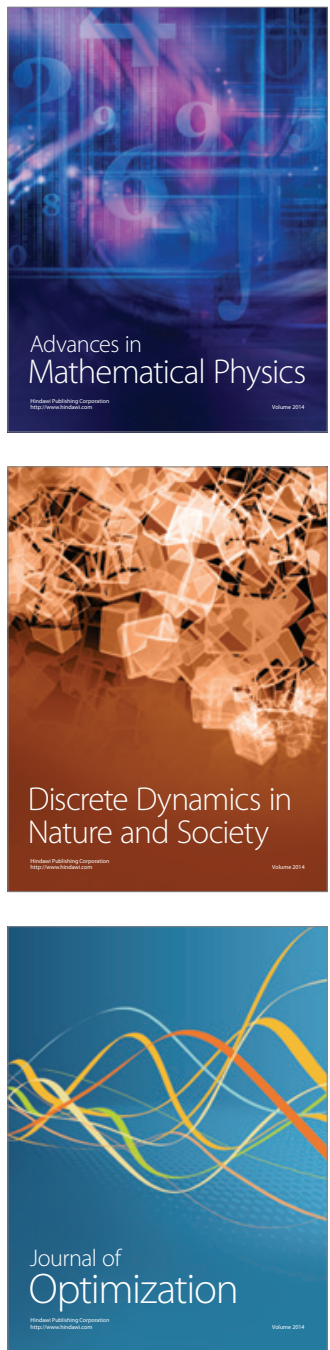